\title{
Adaptive UEP and Packet Size Assignment for Scalable Video Transmission over Burst-Error Channels
}

\author{
Chen-Wei Lee, ${ }^{1}$ Chu-Sing Yang, ${ }^{1}$ and Yih-Ching Su ${ }^{2}$ \\ ${ }^{1}$ Department of Computer Science and Engineering, National Sun Yat-Sen University, Kaohsiung 804, Taiwan \\ ${ }^{2}$ Department of Information Engineering, I-Shou University, Kaohsiung 840, Taiwan
}

Received 28 February 2005; Revised 14 August 2005; Accepted 12 September 2005

This work proposes an adaptive unequal error protection (UEP) and packet size assignment scheme for scalable video transmission over a burst-error channel. An analytic model is developed to evaluate the impact of channel bit error rate on the quality of streaming scalable video. A video transmission scheme, which combines the adaptive assignment of packet size with unequal error protection to increase the end-to-end video quality, is proposed. Several distinct scalable video transmission schemes over bursterror channel have been compared, and the simulation results reveal that the proposed transmission schemes can react to varying channel conditions with less and smoother quality degradation.

Copyright @ 2006 Hindawi Publishing Corporation. All rights reserved.

\section{INTRODUCTION}

Bit errors and packet losses are common throughout the wired/wireless Internet. They severely influence the quality of delay-sensitive multimedia applications. In the current Internet architecture, the application must react to the perceived congestion in the network. The availability of simple and efficient loss models enhances the ability of applications to react. A number of studies have shown that loss patterns exhibit a finite dependence in time $[1,2]$. The most generalized model of quasistationary phenomena is a finite-state Markov chain. A two-state Markov model or Gilbert model $[3,4]$ is often used to simulate burst loss patterns over a wired/wireless channel [5-7] because it is simple and effective.

Many authors have proposed scalable video coders for Internet applications [8-11]. Of these, the fine granular scalability (FGS) $[11,12]$ of MPEG-4 is a highly flexible coding technique that can deliver layered video data with precise rate control. The characteristics of FGS are considered to be advantages in error-prone heterogeneous transmission environments, such as mobile video-on-demand systems. The idea of combining scalable coding with unequal error protection (UEP) has already been proven to result in transmission schemes that exhibit graceful degradation [13-18]. Several adaptive UEP assignment schemes over distinct transmission conditions have been described [13-18]; however, the inherent tradeoff between the selection of packet size and the consequent packet error rate (PER) on a multiaccess channel has not been mentioned. For wireless links, the dominant error mechanism is bit error. A larger packet corresponds to higher probabilities of unrecoverable bit errors and packet loss. However, a smaller packet has a higher header overhead. In the context of streaming video, a lossy channel exacerbates the problem if the packet size is not adaptive. The authors of [19] sought to find optimal bit allocation in FECbased video, but they only considered Internet video without wireless video (bit error). In [20], a dynamic packet size mechanism in wireless networks was proposed, but the problems associated with burst-error channel and the adaptation of video quality have not been addressed.

This work investigates a video transmission scheme, which combines a scalable video coder with unequal error protection (UEP) and adaptive packet size (APS) assignment, to cope with the sometimes rapidly varying transmission conditions over wired or wireless Internet connections. The approach does not require any support from the network. It can be employed in any packet-oriented network.

This paper is organized as follows. Section 2 introduces the burst-error channel model; several functions have been derived to calculate the packet error rate from bit error rate. Section 3 describes how the hierarchically encoded video stream is packetized; protected by unequal error protection, and transmitted to the receiver. The complete analytical model in a scalable video stream is formulated. Section 4 presents the adaptive assignment algorithms for scalable video transmission over a burst-error channel. Section 5 evaluates the performance of distinct scalable video transmission schemes over a burst-error channel. Finally, Section 6 draws conclusions. 


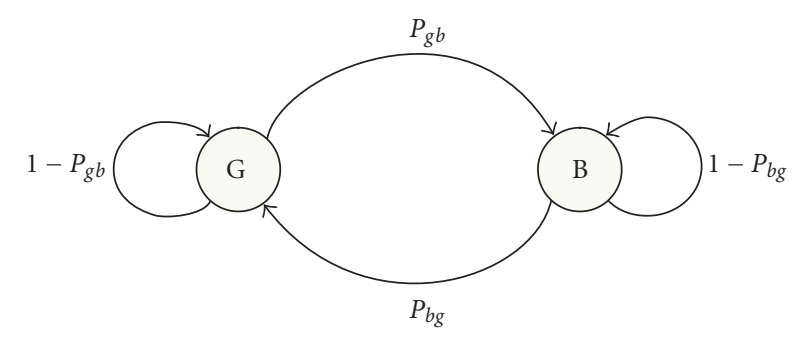

FIGURE 1: Gilbert channel model.

\section{CHANNEL MODEL}

A two-state Markov model or a Gilbert model is used to simulate burst loss patterns over a wired/wireless channel. The two states of the model are denoted by $\mathrm{G}$ and $\mathrm{B}$ (Figure 1). In state $\mathrm{G}$, a bit is received correctly, while in state B, a bit is lost. This model is described by an average bit error rate $P_{b}$ and an average burst bit error length $L_{b}$. The transition probabilities $P_{g b}$ and $P_{b g}$ can be easily computed using $P_{b}$ and $L_{b}$ :

$$
\begin{gathered}
P_{b g}=\frac{1}{L_{b}}, \\
P_{g b}=P_{b g} \frac{P_{b}}{1-P_{b}} .
\end{gathered}
$$

Packet loss patterns can be determined using the packet error rate $P_{B}$ (PER) and average burst packet loss length $L_{B}$, known in advance. $P_{B}$ and $L_{B}$ are calculated as follows:

$$
\begin{gathered}
P_{B}=1-\left(\left(1-P_{b}\right) \cdot\left(1-P_{g b}\right)^{\tau-1}\right), \\
P_{G B}=1-\left(1-P_{g b}\right)^{\tau}, \\
P_{B G}=P_{G B} \cdot \frac{\left(1-P_{B}\right)}{P_{B}}, \\
L_{B}=\frac{1}{P_{B G}},
\end{gathered}
$$

$\tau$ denotes packet length in bits.

Figure 2 shows the relationships among $P_{B}$ (PER), $P_{b}$ (BER), and $L_{b}$. In Figure 2(a), at $P_{b}=10^{-3}, P_{B}$ decreases as $L_{b}$ increases, and a shorter packet corresponds to a smaller $P_{B}$. Figure 2(b) shows that $P_{B}$ increases following the rise of $P_{b}$ in a specific burst state (such as $L_{b}=70$ ).

Figure 3 presents the relationship between $P_{B}(\mathrm{PER})$ and packet size. In Figure 3, at $L_{b}=20, P_{B}$ increases with packet length, and a smaller $P_{b}$ (BER) corresponds to a smaller $P_{B}$.

\section{PACKETIZATION SCHEME}

The packetization scheme employed herein shows how the hierarchically encoded video stream is packetized, protected by unequal error protection, and transmitted to the receiver. As illustrated in Figure 4, bit streams of all layers are interleaved into one block of packets (BOP), and the transmitted packets are the rows of BOP. Notably, the source data with

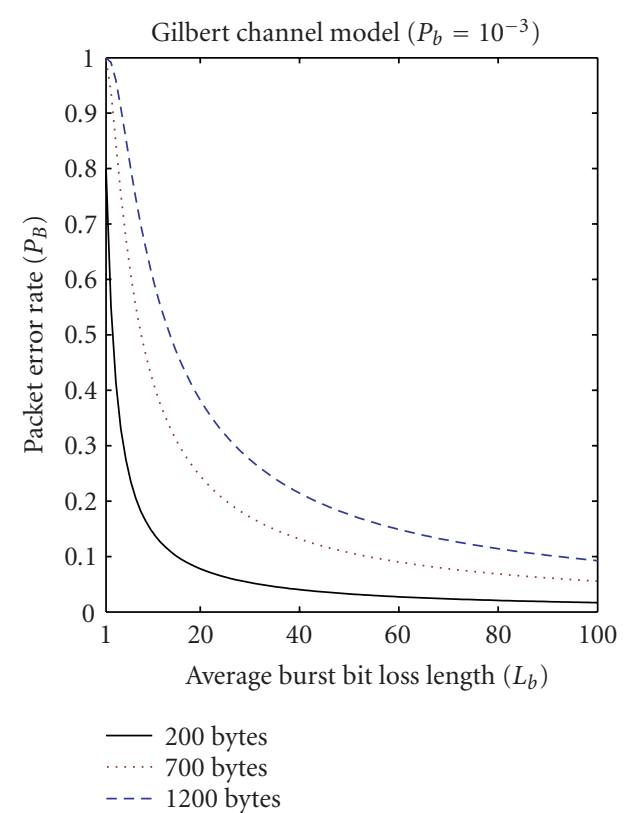

(a)

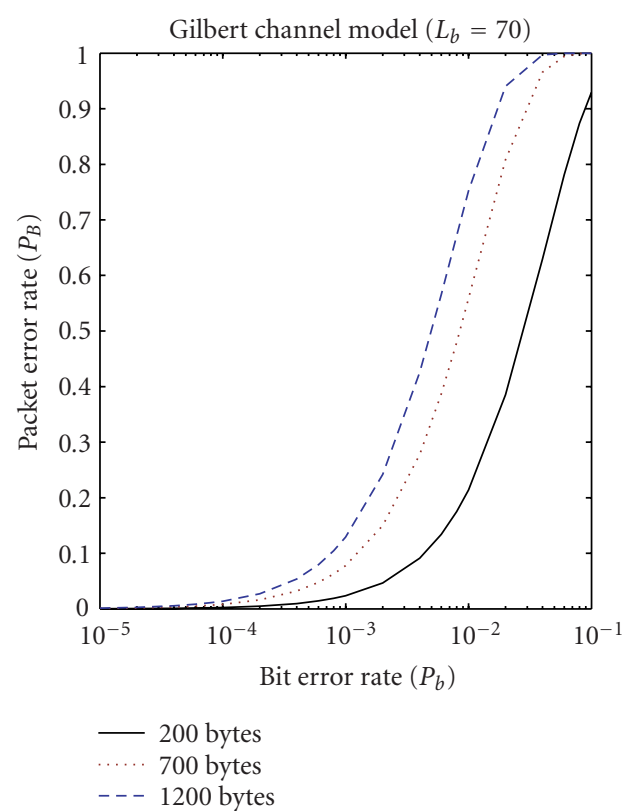

(b)

Figure 2: Relationships among $P_{B}$ (PER), $P_{b}$ (BER), and $L_{b}$.

length $r_{i}$ in layer $i(i=0 \sim l$, where layer 0 is the base layer) are grouped into $k_{i}$ packets (column width $=s_{i}$ ), and the remaining $n-k_{i}$ packets ( $n=$ number of packets) in the BOP are filled with channel coding redundancy. Therefore, $k_{i}$ specifies the protective level of layer $i . s_{h}$ represents the length of the packet header. The BOP buffer size $r$ is assumed to be fixed to satisfy the buffer and delay constraints for real-time communication. If the number of packets $n$ is known, then the packet size $s=r / n$ in Figure 4 is also known. Now, the 


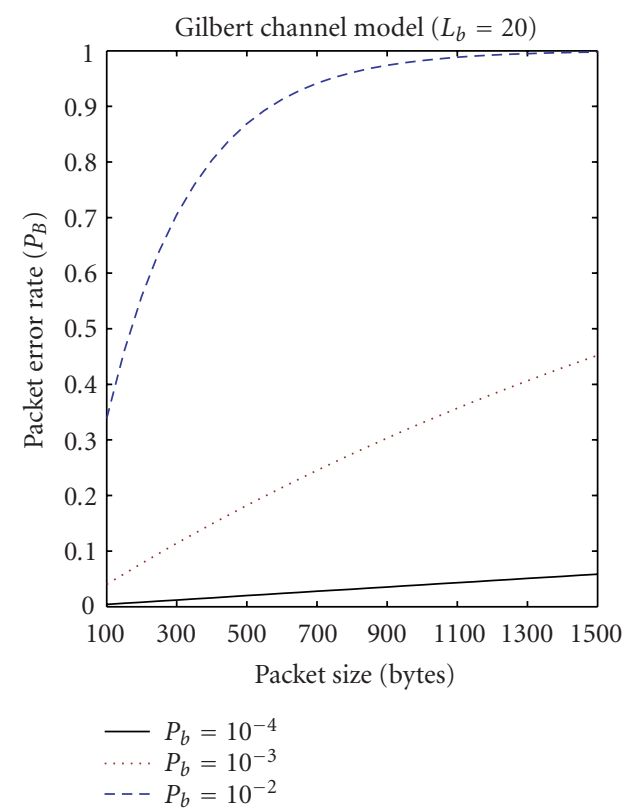

FIGURE 3: Relationship between $P_{B}$ (PER) and packet size.

first FEC assignment constraint obtained from the BOP data structure in Figure 4 is

$$
s=s_{h}+\sum_{i=0}^{l} s_{i}=s_{h}+\sum_{i=0}^{l} \frac{r_{i}}{k_{i}} .
$$

The video data are assumed to be transmitted with constant bit rate, and each group of pictures (GOP) can be packed into a fixed number of BOPs. In this work, $1 \mathrm{GOB}=$ $1 \mathrm{BOP}$. The dependency of data across stream layers is such that occasional error propagation can greatly reduce the quality of reception. Therefore, the intrinsic importance priority of the layered or scalable video data explicitly requires an unequal error protection scheme, yielding another restriction for FEC assignment:

$$
0 \leq k_{0} \leq k_{1} \leq \cdots \leq k_{l} \leq n .
$$

Packet loss over a Gilbert channel can be modeled as a renewal error process [4]. Restated, the lengths of consecutive intererror intervals (also called gaps) are independently and identically distributed. Following [4], let $g(i)$ be the probability that the gap length is $i-1, g(i)=\operatorname{Pr}\left(0^{i-1} 1 \mid 1\right)$, where " 1 " represents a lost packet and $0^{i-1}$ denotes $i-1$ consecutively received packets. Similarly, let $G(i)$ be the probability that a gap length exceeds $i-1: G(i)=\operatorname{Pr}\left(0^{i-1} \mid 1\right)$. Then the probability $R(m, n)$ that $m-1$ packet losses occur in the next $n-1$ packets following an error can be computed by recurrence [4]. Thus,

$$
R(m, n)= \begin{cases}G(n), & m=1, \\ \sum_{i=1}^{n-m+1} g(i) R(m-1, n-i), & 2 \leq m \leq n .\end{cases}
$$

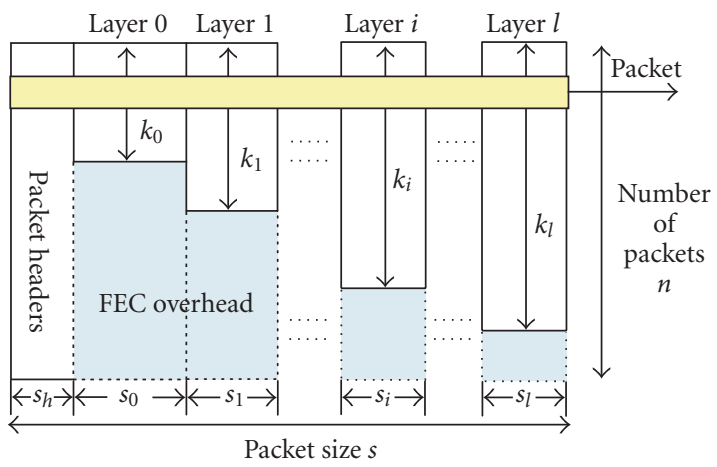

Figure 4: Data structure of BOP (block of packets).

Finally the probability of $m$ lost packets within a block of $n$ packets or $P(m, n)$ is [4]

$$
P(m, n)= \begin{cases}\sum_{i=1}^{n-m+1} P_{B} G(i) R(m, n-i+1), & 1 \leq m \leq n, \\ 1-\sum_{m=1}^{n} P(m, n), & m=0 .\end{cases}
$$

$P_{B}$ in (10) is the average packet loss rate of a Gilbert model.

Combining the BOP data structure with the Gilbert model, renewal error process yields the following complete analytical model of the expected received quality of a BOP in a scalable video stream, as in the authors' earlier work $[13,21,22]$ :

$$
\begin{aligned}
& \eta=\sum_{i=0}^{l+1} \sum_{m=n-k_{i}+1}^{n-k_{i-1}} P(m, n)(\underbrace{\sum_{j=0}^{i-1} \chi_{j}}_{\begin{array}{c}
\text { perfectly } \\
\text { reconstructed } \\
\text { quality }
\end{array}}+\underbrace{\sum_{j=1}^{n-m+1} \frac{\rho_{j} R(m, n-j+1)}{P(m, n)} \phi_{i j}}_{\begin{array}{c}
\text { partially } \\
\text { reconstructed } \\
\text { quality }
\end{array}}), \\
& k_{-1}=0, k_{l+1}=n+1,
\end{aligned}
$$

where

(i) $\eta$ : expected received quality for a BOP;

(ii) $\chi_{i}$ : incremented quality that layer $i$ is received correctly;

(iii) $\phi_{i j}$ : residual quality associated with the correct reception of the first $j-1$ packets of layer $i$;

(iv) $\rho_{j}$ : probability that the first error occurs in packet $j$.

For real-time processing or embedded system environment, an efficient version of the analytical model, which ignores the partially reconstructed quality term in (12), is given to mitigate the computing horsepower requested by adaptive assignment task [13]:

$$
\eta \approx \sum_{i=0}^{l+1} \sum_{m=n-k_{i}+1}^{n-k_{i-1}} P(m, n) \cdot \sum_{j=0}^{i-1} \chi_{j}
$$




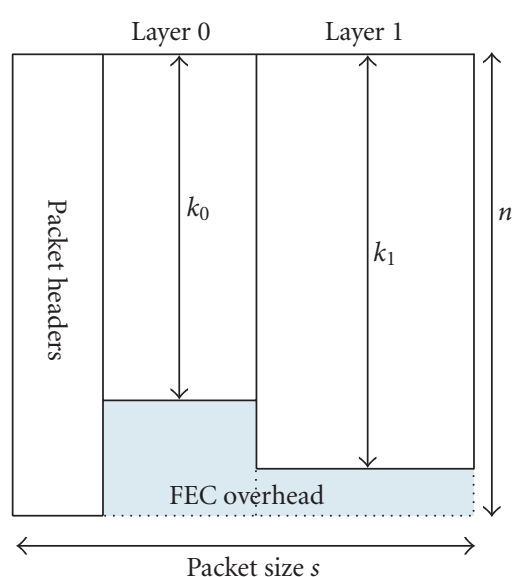

(a) Good channel condition

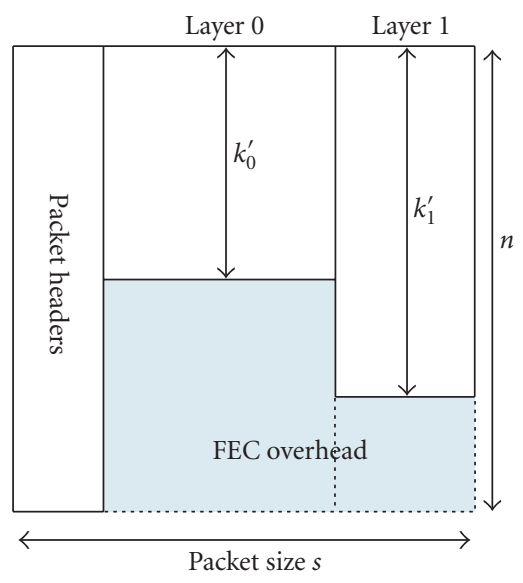

(b) Bad channel condition

FIGURE 5: BOP structure for AUEP+FPS.

\section{ADAPTIVE ASSIGNMENT SCHEME}

This section elucidates the adaptive assignment algorithms for scalable video transmission over burst-error channels. UEP and packet size are initially set by one of four methods:

(1) FUEP (fixed UEP)+FPS (fixed packet size),

(2) AUEP (adaptive UEP)+FPS,

(3) FUEP + APS (adaptive packet size),

(4) AUEP + APS.

\section{$A U E P+F P S$}

Figure 5 shows the BOP structure for AUEP+FPS. Forward error correction (FEC) protection is adaptive but the packet size and the number of packets are fixed. When the channel condition is poor, it typically requires an increasing FEC protection ratio (Figure 5(b)). However, determining how much FEC protection should be added is an optimization problem.

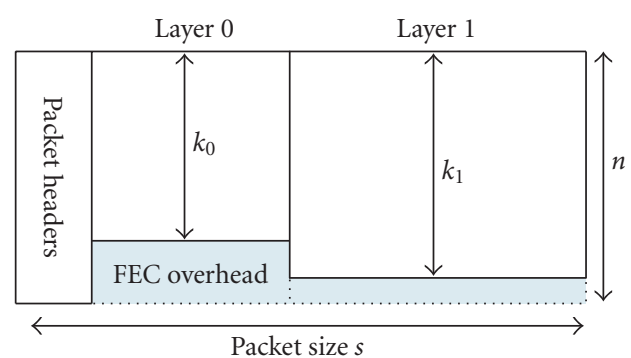

(a) Good channel condition

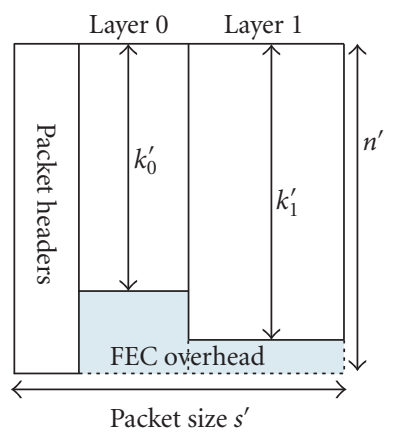

(b) Bad channel condition

FIgURE 6: BOP structure for FUEP + APS.

The adaptive UEP assignment problem can be solved by determining the argument $K$ that maximizes the received video quality $\eta$ according to (12):

$$
\arg \max _{K}\{\eta(K)\}
$$

where $K=\left(k_{0}, k_{1}, k_{2}, \ldots, k_{l}\right)^{t}$.

In (13), the expected quality $\eta$ is explicitly expressed as the function of $K$. In solving (13), the previously described constraints (7) and (8) must be applied. Rearranging (8) yields the rate of layer $l, r_{l}=k_{l}\left(s-s_{h}-\sum_{i=0}^{l-1}\left(r_{i} / k_{i}\right)\right)$, and $r_{l}$ determines the value of $\chi_{l}$. The dynamic programming technique is used to search for the global maximum of quality $\eta$ in (13).

Therefore, when a BOP is transmitted, first the packet size $s$ (FPS) is determined and $P_{B}$ is dynamically derived from (3) with a varying channel condition $\left(P_{b}, L_{b}\right)$. Then, the FEC protection ratio for each layer in a BOP can be determined from (13).

$F U E P+A P S$

Figure 6 presents the variation in the BOP structure under various channel conditions for FUEP + APS. The FEC protection ratio is fixed and the packet size is adaptive. A smaller packet size is typically required to reduce PER when the channel condition becomes bad (Figure 6(b)), but increasing the number of packets increases the header overheads. 


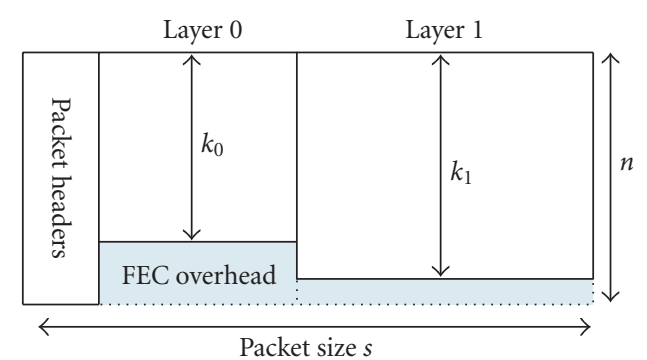

(a) Good channel condition

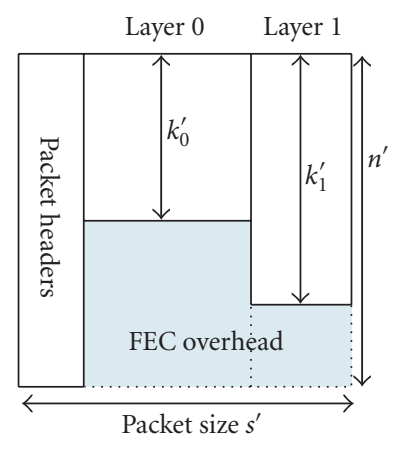

(b) Bad channel condition

FIgURE 7: BOP structure for AUEP + APS.

The adaptive packet size assignment problem is to find $n$ (number of packets) that maximizes the received video quality $\eta$ :

$$
\arg \max _{n}\{\eta(n)\} .
$$

After $n$ is found, the adaptive packet size can be determined, since $s=r / n$, where $r$ is the BOP buffer size. Each adaptive packet size herein was derived and applied for each BOP unit. The packet size was constrained to satisfy $s \leq 1500$ bytes because of the MTU (maximum transmission unit).

\section{$A U E P+A P S$}

The AUEP and APS together yield the variation of the BOP structure under different channel conditions in Figure 7. Packet size and FEC protection ratio must be concerned simultaneously. The optimization problem can be rewritten as

$$
\arg \max _{n, K}\{\eta(n, K)\} .
$$

In solving (15), the aforementioned constraints equations (7) and (8) must be observed.

\section{SIMULATION RESULTS}

This section demonstrates the performance evaluation of distinct scalable video transmission schemes over the bursterror channel discussed above. The common test conditions for all of video streams used in this work are as follows.

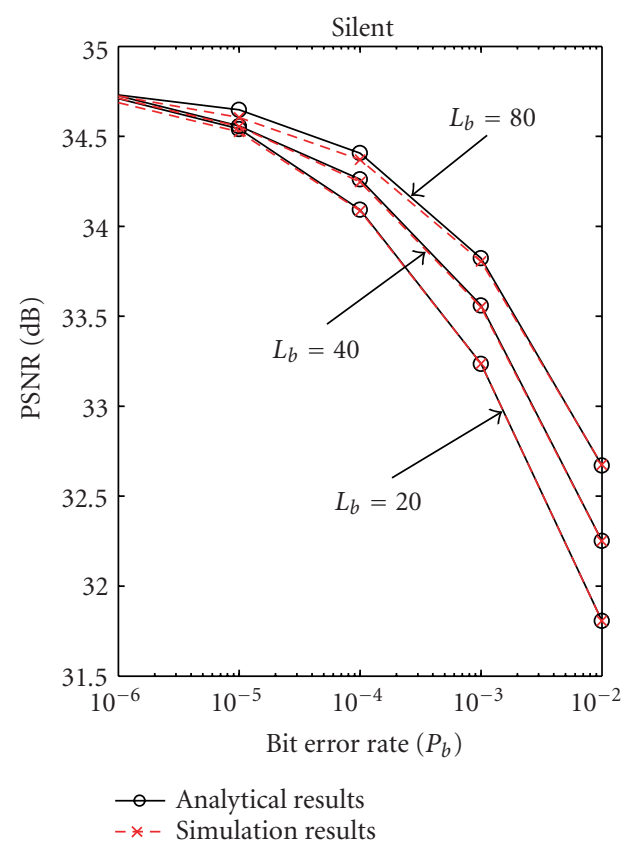

FIGURE 8: Comparison of the analytical and the simulated PSNR values obtained using the AUEP + APS scheme.

(i) Frame resolution $=$ CIF format $-352 \times 288$ pixels.

(ii) Constant stream rate $=256$ Kbps.

(iii) $1 \mathrm{GOP}=1$ intraframe accompanied with 14 interframes, and frame rate $=15 \mathrm{fps}$.

(iv) Sequence length $=9$ GOPs.

(v) MPEG-4 FGS method is adopted to generate scalable video streams. Each MPEG-4 FGS video stream comprises one base layer and one enhancement layer.

(vi) Packet header length: 40 bytes (IP/UDP/RTP).

Figure 8 compares the analytical and simulated PSNR values obtained using the AUEP + APS scheme (15) at various bit error rates $P_{b}$. In this example, the test video sequence is "silent," and the burst bit error lengths $L_{b}$ for the three curves are 20,40 , and 80 , respectively. As presented in Figure 8 , the simulation results are very close to the analytical results, confirming the correctness of (15).

\section{$A U E P+F P S$}

Initially, the AUEP+FPS scheme is being considered. The performance of the EEP (equal error protection) scheme, the nonadaptive UEP scheme, and the proposed adaptive UEP scheme under various channel conditions with FPS is compared.

Figure 9 compares the performance of the AUEP scheme with some other fixed error protection schemes, using the test sequences "Foreman" and "News"; the channel-related parameters are $s=600$ bytes, $L_{b}=20$, and $P_{b}=0 \sim 10^{-3}$. The actions of various error protection schemes are identified clearly under various channel conditions, using not only the proposed AUEP scheme but three other typical fixed error protection schemes (FUEP)—(1) protecting base layer 

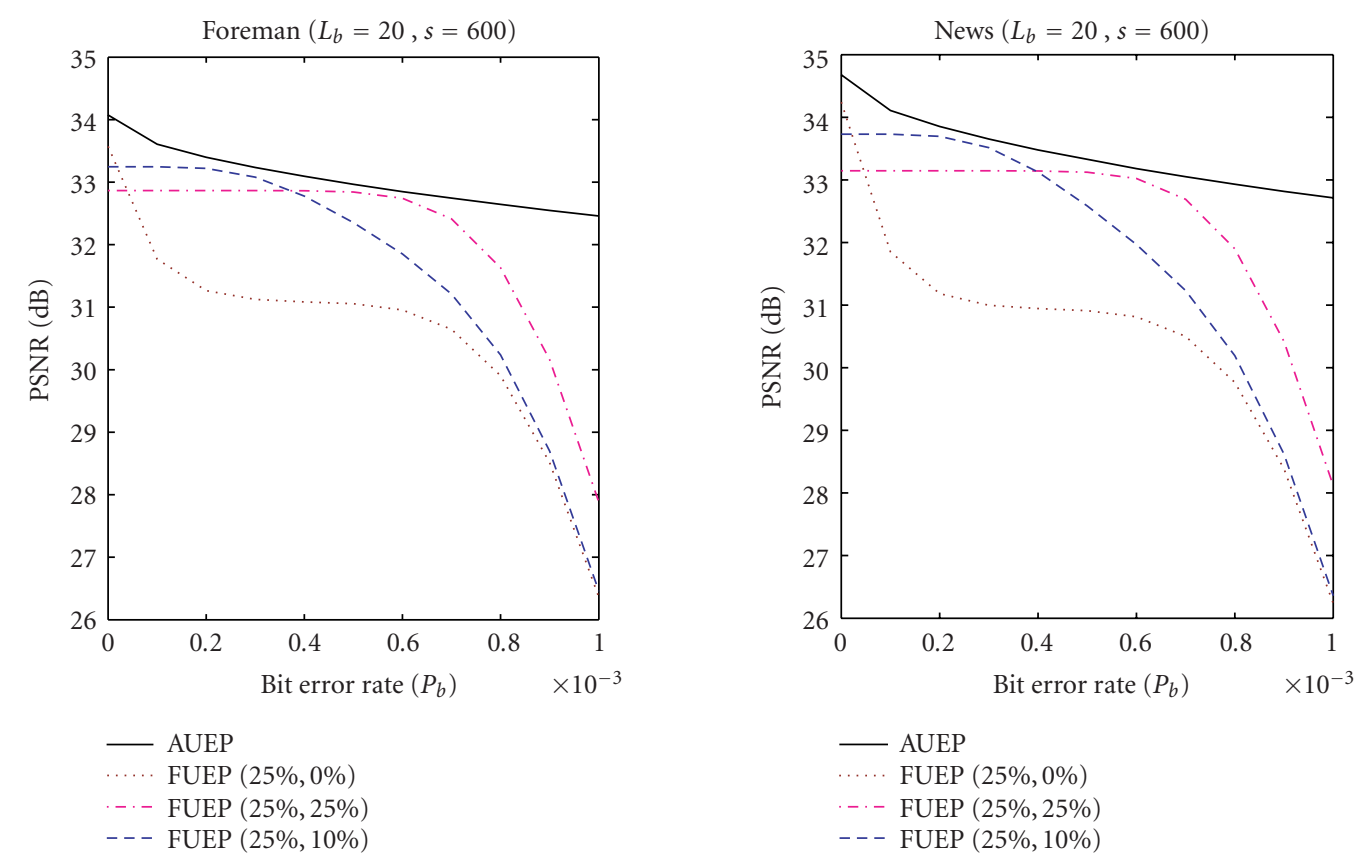

(a) FUEP schemes with low protection ratios
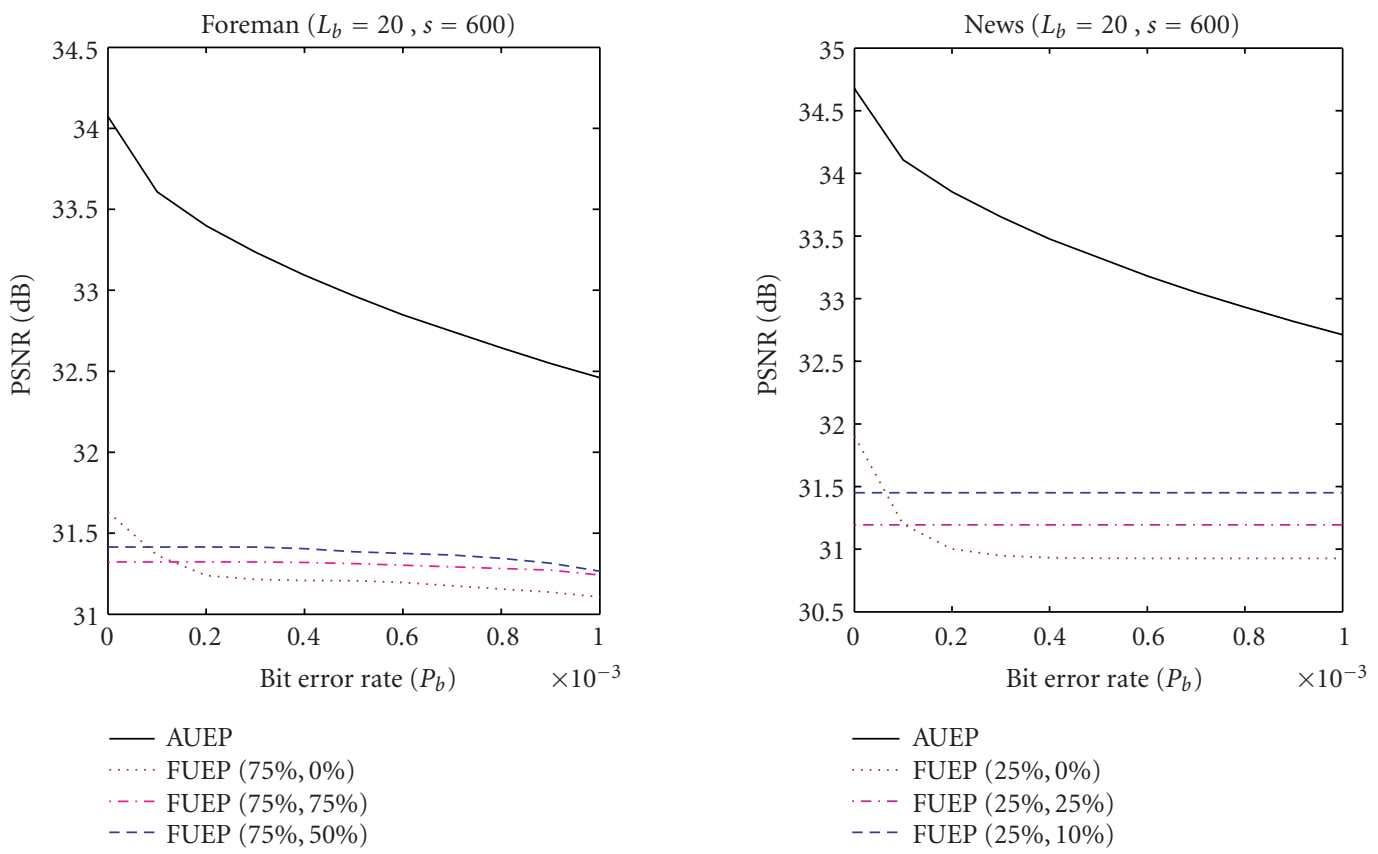

(b) FUEP schemes with high protection ratios

FIGURE 9: Comparison of performance of proposed adaptive UEP scheme (AUEP) and three other fixed error protection schemes (FUEP) with (a) low protection ratios and (b) high protection ratios.

only with fixed protection overhead; (2) protecting all layers with fixed and equal protection overheads; (3) protecting all layers with fixed but unequal protection overheads.

More specifically, the fixed protection ratios for FUEP are classified as low (Figure 9(a)) or high (Figure 9(b)). The percentages in parentheses in the legends in Figure 9 separately represent the protection ratios for the base layer (left) and the enhancement layer (right). Figure 9 shows that only AUEP can effectively adapt to the channel conditions and react to the poorer conditions with smoother quality 

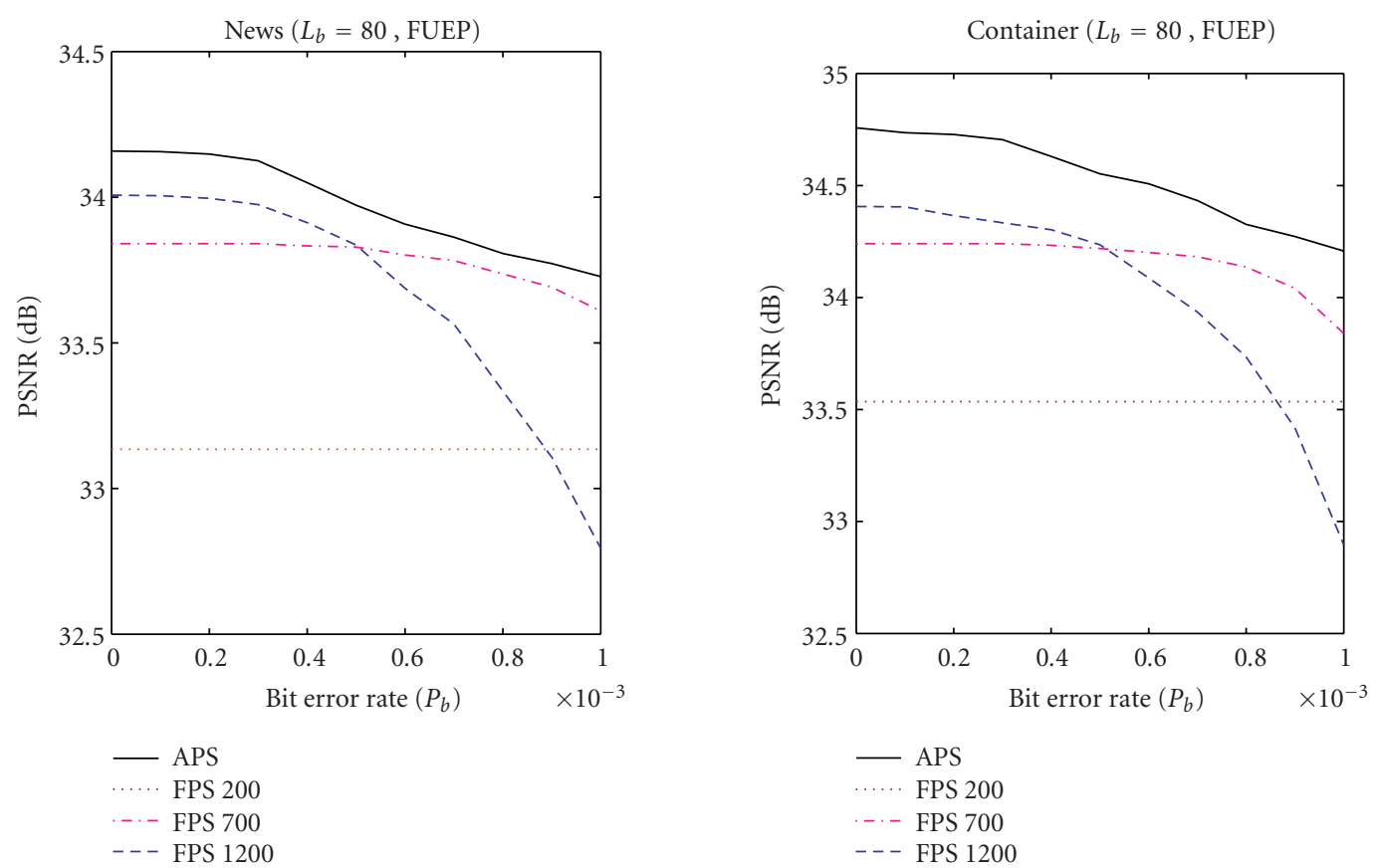

(a) Low FUEP $(25 \%, 10 \%)$
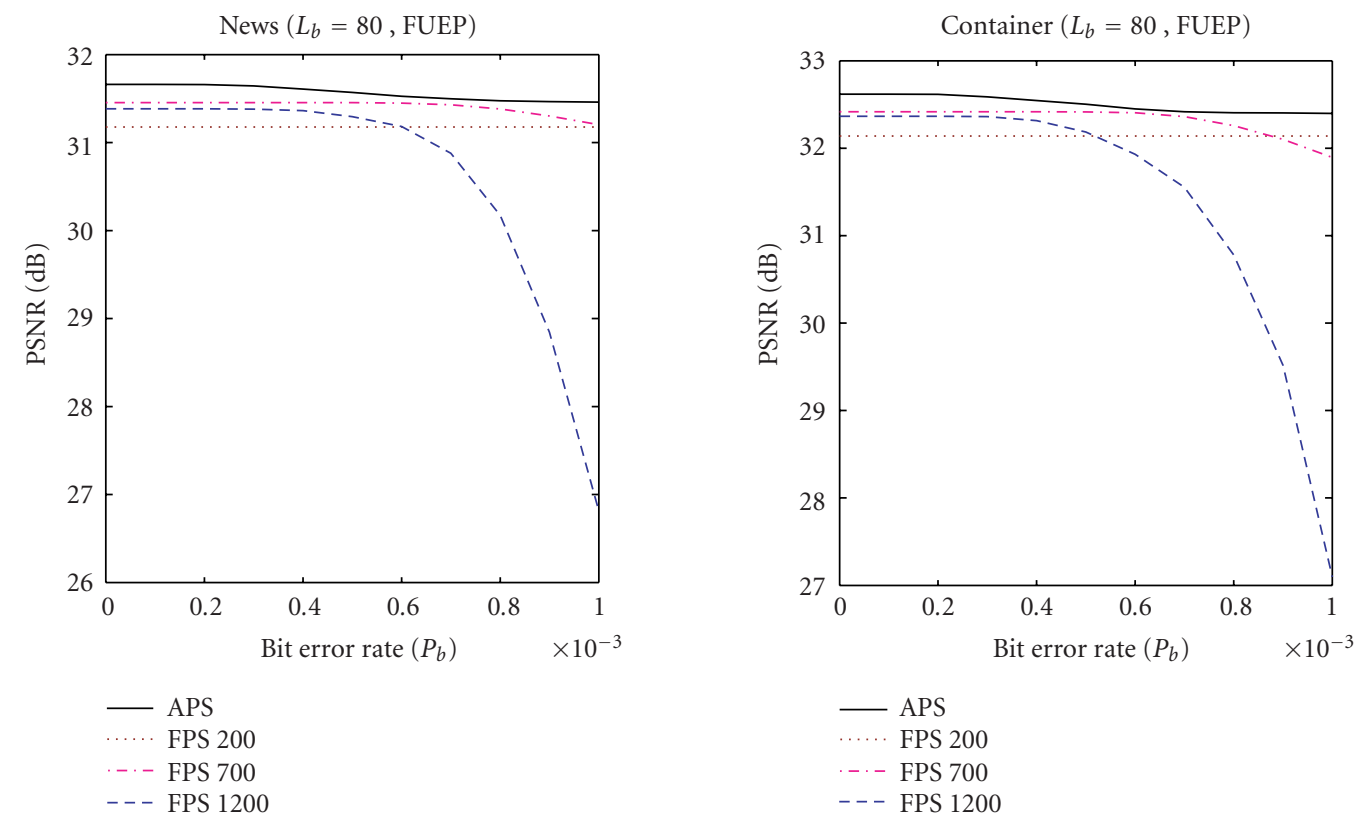

(b) High FUEP (75\%, 50\%)

FIGURE 10: Comparison of performance of proposed adaptive packet size scheme and three other fixed packet size schemes at (a) low protection ratios and (b) high protection ratios.

degradation. In contrast, regardless of the allocation of the protection overhead, the other three nonadaptive schemes (FUEP) will be associated with either too little protection under bad channel conditions or too much protection under good channel conditions. The outcome is severe quality degradation.

\section{$F U E P+A P S$}

Then, the performance of several FPS schemes and the proposed APS scheme under various channel conditions with FUEP is compared. Figure 10 compares the performance of the proposed APS scheme with some other FPS schemes, 

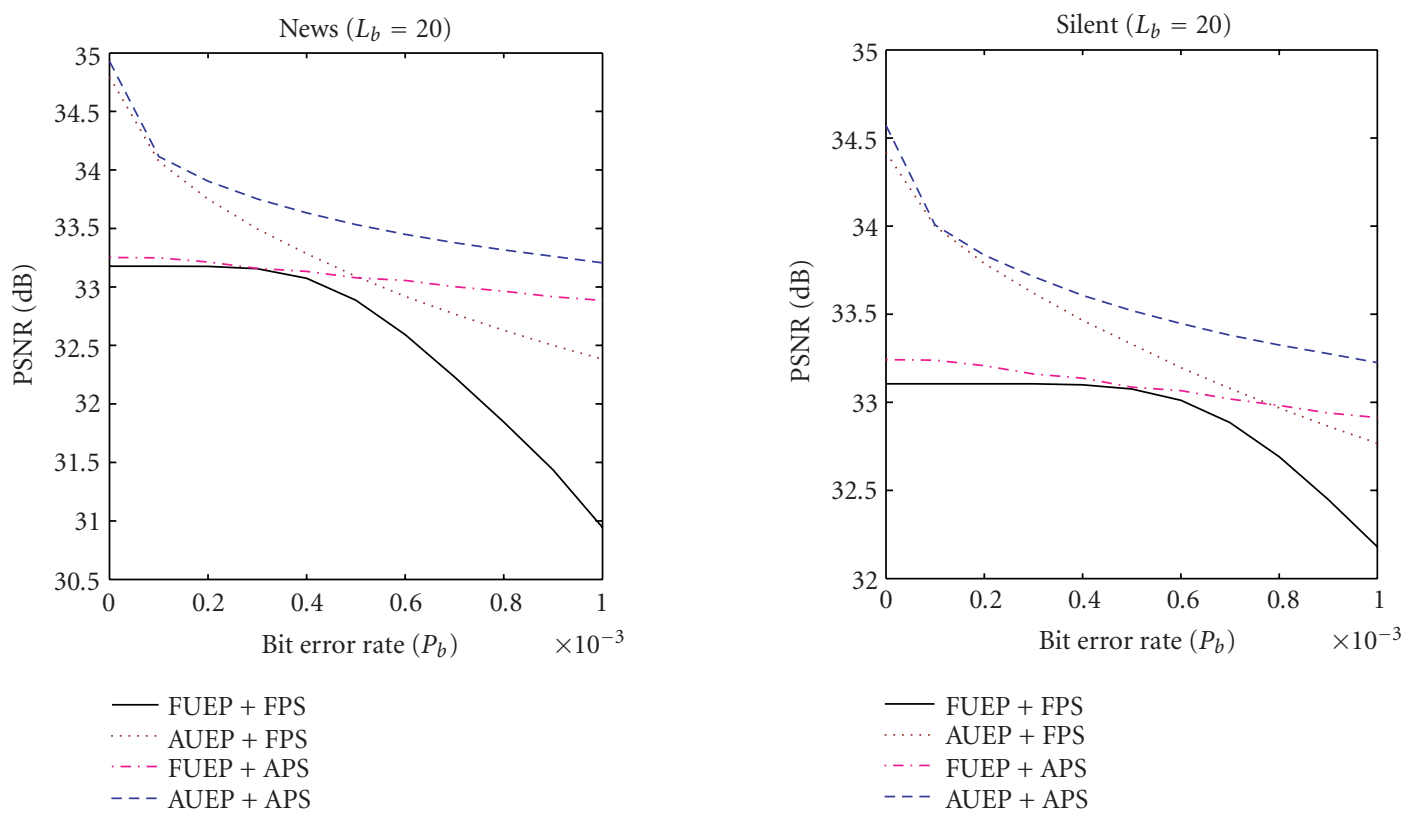

FIGURE 11: Comparison of performance of four proposed assignment schemes. The fixed parameters are FUEP (40\%, 20\%) and FPS (800 bytes).

using the test sequences "News" and "Container." The channel parameters are $L_{b}=80$ and $P_{b}=0 \sim 10^{-2}$. The adaptive UEP scheme was not considered herein. For convenience, fixed 200 bytes are labeled FPS200, and so on. Specifically, the fixed protection ratios are classified into two levelslow protection ratio (Figure $10(\mathrm{a})$ ) or high protection ratio (Figure 10(b)).

Figure 10 reveals that the length of the packet influences the quality of the received video, and the proposed APS scheme effectively adapts to the various channel conditions. In contrast, regardless of the selected length of the packet, the other three nonadaptive schemes (FPS200/FPS700/FPS1200) yield packets that are either too large with high $P_{B}$ under bad channel conditions or too small with a large header overhead under good channel conditions.

\section{$A U E P+A P S$}

Figure 11 displays the evaluated performance of the four UEP and packet size assignment schemes over a burst-error channel. The test sequences are "News" and "Silent"; the channel parameters are $L_{b}=20$ and $P_{b}=0 \sim 10^{-3}$. The fixed parameters are FUEP (40\%, 20\%) and FPS ( 800 bytes). Figure 11 shows that the three adaptive schemes always outperform the fixed scheme under any channel condition. The protection of FUEP (FEC overhead) is too strong under low BER conditions and the packet size obtained using FPS is too small and the header overhead is high under high BER conditions. Comparing the performance of the proposed approaches shows that the AUEP + APS scheme outperforms all the others.

\section{CONCLUSIONS}

This work develops an analytic model of the effect of the channel bit error rate on the packet error rate, which in turn affects the quality of the streaming video, to increase the end-to-end video quality over the burst-error channel. This work proposes AUEP+FPS, FUEP + APS, and AUEP + APS assignment schemes for scalable video transmission over a burst-error channel. For a given total available bandwidth, content-adaptive plus channel-adaptive assignment of packet size and UEP is achieved simultaneously. Simulation results reveal that the proposed methods can effectively adapt to various channels and react to the poor channel conditions, with a smaller and smoother drop in quality. The approach proposed herein does not require any support from the network, and so can be employed in any packet-oriented network.

\section{REFERENCES}

[1] M. Yajnik, S. Moon, J. Kurose, and D. Towsley, "Measurement and modelling of the temporal dependence in packet loss," in Proceedings of 18th Eighteenth Annual Joint Conference of the IEEE Computer and Communications Societies (INFOCOM '99), vol. 1, pp. 345-352, New York, NY, USA, March 1999.

[2] R. Singh and A. Ortega, "Modeling temporal dependence in packet loss using universal modeling concepts," in Proceedings of 12th International Packet Video Workshop (PV '02), Pittsburgh, Pa, USA, April 2002.

[3] E. N. Gilbert, "Capacity of a burst-noise channel," Bell System Technical Journal, vol. 39, pp. 1253-1265, 1960.

[4] E. O. Elliott, "A model of the switched telephone network for data communications," Bell System Technical Journal, vol. 44, no. 1, pp. 89-109, 1965. 
[5] C. C. Tan and N. C. Beaulieu, "On first-order Markov modeling for the Rayleigh fading channel," IEEE Transactions on Communications, vol. 48, no. 12, pp. 2032-2040, 2000.

[6] C.-Y. Hsu and A. Ortega, "A Lagrangian optimization approach to rate control for delay-constrained video transmission over burst-error channels," in Proceedings of the IEEE International Conference on Acoustics, Speech, and Signal Processing (ICASSP '98), vol. 5, pp. 2989-2992, Seattle, Wash, USA, May 1998.

[7] H. S. Wang and N. Moayeri, "Finite-state Markov channel—a useful model for radio communication channels," IEEE Transactions on Vehicular Technology, vol. 44, no. 1, pp. 163-171, 1995.

[8] B. Kim, Z. Xiong, and W. A. Pearlman, "Low bit-rate scalable video coding with 3-D set partitioning in hierarchical trees (3D SPIHT)," IEEE Transactions on Circuits and Systems for Video Technology, vol. 10, no. 8, pp. 1374-1387, 2000.

[9] F. Wu, S. Li, and Y.-Q. Zhang, "A framework for efficient progressive fine granularity scalable video coding," IEEE Transactions on Circuits and Systems for Video Technology, vol. 11, no. 3, pp. 332-344, 2001.

[10] D. Taubman and A. Zakhor, "Multirate 3-D subband coding of video," IEEE Transactions on Image Processing, vol. 3, no. 5, pp. 572-588, 1994.

[11] W. Li, "Overview of fine granularity scalability in MPEG-4 video standard," IEEE Transactions on Circuits and Systems for Video Technology, vol. 11, no. 3, pp. 301-317, 2001.

[12] ISO/IEC 14496-10, "Information technology-Coding of audio-visual objects-Part 10: Advanced video coding," also ITU-T Recommendation H.264: Advanced video coding for generic audiovisual services, 2003.

[13] Y.-C. Su, C.-S. Yang, and C.-W. Lee, “Optimal FEC assignment for scalable video transmission over burst error channel with loss rate feedback," Signal Processing: Image Communication, vol. 18, no. 7, pp. 537-547, 2003.

[14] G. Wang, Q. Zhang, W. Zhu, and Y. Zhang, "Channel-adaptive unequal error protection for scalable video transmission over wireless channel," in Visual Communications and Image Processing (VCIP '01), vol. 4310 of Proceedings of SPIE, pp. 648655, San Jose, Calif, USA, January 2001.

[15] U. Horn, K. Stuhlmuller, M. Link, and B. Girod, "Robust Internet video transmission based on scalable coding and unequal error protection," Signal Processing: Image Communication, vol. 15, no. 1-2, pp. 77-94, 1999.

[16] T. Stockhammer, "Progressive video transmission for packet lossy channels exploiting feedback and unequal erasure protection," in Proceedings of the International Conference on Image Processing (ICIP '01), vol. 2, pp. 169-172, Rochester, NY, USA, September 2002.

[17] J. Vass and X. Zhuang, "Adaptive and integrated video communication system utilizing novel compression,error control, and packetization strategies for mobile wireless environments," in Proceedings of the 10th International Packet Video Workshop (PV'00), Cagliari, Italy, May 2000.

[18] M. van der Schaar and H. Radha, "Packet-loss resilient Internet video using MPEG-4 fine granular scalability," in Proceedings of the International Conference on Image Processing (ICIP '00), vol. 3, pp. 372-375, Vancouver, BC, Canada, September 2000.

[19] B. Hong and A. Nosratinia, "Rate-constrained scalable video transmission over the Internet," in Proceedings of 12th International Packet Video Workshop (PV'02), Pittsburgh, Pa, USA, April 2002.
[20] T. Vu, D. Reschke, and W. Horn, "Dynamic packet size mechanism (DPSM) for multimedia in wireless networks," in Proceedings of Multimediale Informations- und Kommunikationssysteme (MIK'02), pp. 667-674, Erfurt, Germany, SeptemberOctober 2002.

[21] Y.-C. Su, C.-S. Yang, and C.-W. Lee, "The analysis of packet loss prediction for Gilbert-model with loss rate uplink," Information Processing Letters, vol. 90, no. 3, pp. 155-159, 2004.

[22] C. S. Yang, C. W. Lee, and Y. C. Su, "Adaptive packet size assignment for scalable video transmission over burst error channel," in Proceedings of the 10th International Conference on Distributed Multimedia Systems (DMS '04), pp. 99-103, San Francisco, Calif, USA, September 2004.

Chen-Wei Lee was born in Nantou, Taiwan, on January 10, 1975. He received the B.S. degree in electrical engineering and M.S. degree in computer science and engineering from the National Sun Yat-Sen University, Kaohsiung, Taiwan, in 1997 and 1999, respectively. Currently, he is a Ph.D. student in the Department of Computer Science and Engineering at the National Sun Yat-Sen University. His research interests include multimedia communication and error control coding.

Chu-Sing Yang received the B.S. degree in engineering science, and the M.S. and Ph.D. degrees in electrical engineering from the National Cheng Kung University, Taiwan, in 1976, 1984, and 1987, respectively. He joined the faculty of the Department of Electrical Engineering, National Sun YatSen University, Taiwan, as an Associate Professor in 1988. Since 1993, he has been a Professor in the Department of Computer

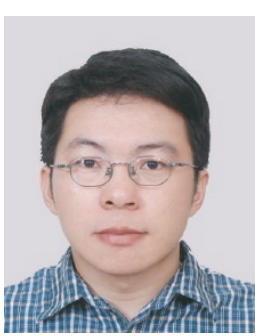
Science and Engineering, National Sun Yat-Sen University. He was a Chair of the Department of Computer Science and Engineering, National Sun Yat-Sen University, from August 1995 to July 1999. He was a Director of the Computer Center, National Sun Yat-Sen University, from August 1998 to October 2002. He was a Program Chair of ICS-96, and Program Cochair of ICPP-2003. His research interests include multimedia communication, parallel and distributed systems, scalable web servers, and wireless communication.

Yih-Ching Su received the B.S. degree in electrical engineering from the National Taiwan University of Science and Technology in 1992, the M.S. degree in biomedical engineering from the National Cheng Kung University in 1995, and the Ph.D. degree in computer science and engineering from the National Sun Yat-Sen University in 2003. He is an Assistant Professor in the Department of Computer Science and Information Engineering in the I-Shou University, Taiwan. His research interests are in all aspects of multimedia communication systems, with special emphasis on video coding, robust video transmission, and multimedia system-on-a-chip (MSOC). 